\title{
Space Charge Frequency Shifts of the Cyclotron Modes in Multi-Species Ion Plasmas
}

\author{
M. Affolter, F. Anderegg, C. F. Driscoll
}

Department of Physics, University of California at San Diego, La Jolla, CA 92093, USA

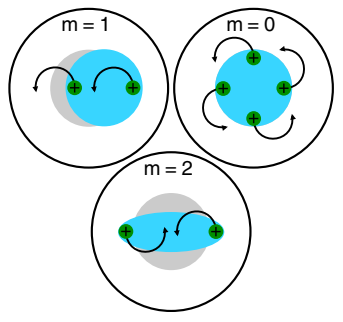

\begin{abstract}
Shifts of the cyclotron frequency away from the "bare" cyclotron frequency are observed to be proportional to the total ion density through the $E \times B$ rotation frequency, and to the relative concentration of each ion species, in quantitative agreement with analytic theory. These shifts are measured at small excitation amplitudes on the typical center-of-mass $m=1$ mode, and also on cyclotron modes with $m=$ 0 and $m=2$ azimuthal dependence. The frequency spacing between these modes is proportional to the rotation frequency of the ion cloud, which is controlled and measured using a "rotating wall" and laser-induced fluorescence. These cylindrical
\end{abstract} ion plasmas consist of $\mathrm{Mg}^{+}$isotopes, with $\mathrm{H}_{3} \mathrm{O}^{+}$and $\mathrm{O}_{2}{ }^{+}$impurities. It is observed that the shift in the $m=1$ cyclotron frequency is larger for the minority species ${ }^{25} \mathrm{Mg}^{+}$and ${ }^{26} \mathrm{Mg}^{+}$, than for the majority species ${ }^{24} \mathrm{Mg}^{+}$. A simple center-of-mass model is presented, which is in quantitative agreement with these results. It is also shown that this model interprets and expands the intensity dependent calibration equation, $(M / q)=A / f+B / f^{2}+C I / f^{2}$.

Keywords: Plasma, Non-neutral, Cyclotron mode, Mass spectrometry, Space charge, Calibration equation, Frequency shift

Received: 29 July 2014/Revised: 17 October 2014/Accepted: 21 October 2014/Published online: 2 December 2014

\section{Introduction}

E lectric fields and collective effects often limit the accuracy of Fourier transform ion cyclotron resonance mass spectrometry (FTICR-MS), by shifting the observed frequencies away from the "bare" cyclotron frequencies $2 \pi F_{c}^{(s)}=q_{s} B_{z} / M_{s}$ for each species $s$. The electric fields result from applied trap potentials, from ion space charge, and from image charges on the trap wall. These shifts must be understood in order to accurately convert the measured frequencies to a mass-tocharge ratio $(M / q)_{s}$. This conversion is typically done using one of the several calibration equations [1], which account for the space charge electric field and its influence on the cyclotron frequency.

In this paper, the shift of the cyclotron frequencies attributable to these electric fields and collective effects are experimentally investigated for several cyclotron modes varying as $\cos \left(m \theta-2 \pi f_{m}{ }^{(s)} t\right)$. We describe the frequency shift of the typical $m=1$ center-of-mass mode, with additional information on these frequency shifts from the rarely observed radial "breathing" $m=0$ mode[2-4] and "elliptical" $m=2$ mode [5, 6]. These experiments are conducted in the limit of small

Correspondence to: M. J. Affolter; e-mail: maffolter@physics.ucsd.edu excitation amplitude, where the radius of excitation $\Delta_{S}$ is small compared with the plasma radius $R_{p}$. In this limit, the cyclotron modes can be modeled as surface waves.

Experimental results are in close agreement with an axisymmetric $k_{z}=0$ surface wave model[6-9], which predicts $m$ dependent shifts in the cyclotron resonances for species $s$. For radially uniform plasmas, the theory predicts

$f_{m}^{(s)}-F_{c}^{(s)}=\left[(m-2)+\delta_{s}\left(1-\mathscr{R}_{m}\right)\right] f_{E}^{S C}$.

That is, the shifts are proportional to the plasma $E \times B$ rotation frequency $f_{E}{ }^{S C} \equiv e n_{0} / 4 \pi \epsilon_{0} B$ arising from the ion space charge electric field, with a correction proportional to the relative charge fraction $\delta_{s}$ of that species. Here, $\mathscr{R}_{m}$ is a correction attributable to wall image charges. For $m \geq 1$, the wall image charge correction is $\mathscr{R}_{m} \equiv\left(R_{p} / R_{w}\right)^{2 m}$, but $\mathscr{R}_{m} \equiv 0$ for $m=0$. This model neglects frequency shifts from trap potentials, which are negligible in these experiments.

In FTICR-MS, though, frequency shifts from trap potentials may be significant, and the $m=1$ cyclotron mode is generally viewed as a clump of charge orbiting around the trap center rather than as a surface wave. However, in the limit of $\Delta_{s}<R_{p}$, the center-of-mass motion of this clump and, thus, the frequency shifts are equivalent to the $m=1$ surface wave. These surface wave results can, therefore, be interpreted by a center-of-mass 
clump model. Frequency shifts from trap potentials will be incorporated into this model, which will be used to obtain a calibration equation:

$$
\left(\frac{M}{q}\right)_{s}=\frac{A}{f_{1}^{(s)}}+\frac{B}{\left(f_{1}^{(s)}\right)^{2}}+\frac{C \cdot \delta_{s}}{\left(f_{1}^{(s)}\right)^{2}} .
$$

This calibration equation is similar to the "intensity-dependent" calibration equation $[10,11]$, with $\delta_{s}$ replaced by the received wall signal intensity $I_{s}$, which has been found $[11,12]$ to improve mass accuracies. Parameter $A$ calibrates the trap magnetic field strength, and parameter $B$ corrects for frequency shifts caused by the entire ion cloud and trap potentials. Parameter $C$ arises from collective effects and wall image charges. Put simply, this parameter is a result of the fact that a clump of charge cannot "push on itself," complicated somewhat by the fact that the wall image charge can and does "pull" on the clump.

\section{Experimental}

\section{Methods}

A cylindrical Penning-Malmberg trap is used to confine these multi-species plasmas, with wall radius $R_{w}=2.86 \mathrm{~cm}$, trapping potential $V=180 \mathrm{~V}$, and magnetic field of $B=2.965 \pm 0.002$ Tesla. A sketch of this trap is shown in Figure 1 (left). The plasmas consists of ${ }^{24} \mathrm{Mg}^{+},{ }^{25} \mathrm{Mg}^{+}$, and ${ }^{26} \mathrm{Mg}^{+}$; with $\mathrm{H}_{3} \mathrm{O}^{+}$and $\mathrm{O}_{2}{ }^{+}$impurity ions arising from background gas at a pressure $P \leq 10^{-9}$ Torr. A typical isotopic composition is $\delta_{24}=0.54, \delta_{25}=$ $0.09, \delta_{26}=0.10$, with the remaining $27 \%$ a mixture of $\mathrm{H}_{3} \mathrm{O}^{+}$and
$\mathrm{O}_{2}^{+}$. As with FTICR-MS experiments, no free electrons are contained in the trap.

A "rotating wall" technique [13] utilizes azimuthally rotating wall voltages to maintain the plasma in a near-rigid-rotor equilibrium state throughout the course of the cyclotron measurements. The external torque applied by the rotating wall fields can also compress the plasma, enabling controlled plasma radius $0.3<R_{p}<0.6 \mathrm{~cm}$, with density $1.8 \times 10^{7}<n_{0}<6.2 \times$ $10^{7} \mathrm{~cm}^{-3}$, and measured $E \times B$ rotation frequency $9.0<f_{E}<$ $30.0 \mathrm{kHz}$. The length $L_{p} \sim 10 \mathrm{~cm}$ and total charge $N_{q} \sim 2 \times 10^{8} \mathrm{e}$ remain constant.

Detailed measurements of these $\mathrm{Mg}^{+}$plasmas are obtained from laser-induced fluorescence techniques[14]. That is, a UV laser beam $(\lambda \sim 280 \mathrm{~nm})$, perpendicular to the magnetic field, is frequency-scanned over the Doppler-broadened atomic transition $\left(3 \mathrm{~s}^{2} \mathrm{~S}_{1 / 2}, \mathrm{~m}_{j}=-1 / 2 \rightarrow 3 \mathrm{p}^{2} \mathrm{P}_{3 / 2}, \mathrm{~m}_{j}=-3 / 2\right)$ of each $\mathrm{Mg}^{+}$isotope. The resulting induced fluorescence photons are counted using a photomultiplier tube, and we construct the $\mathrm{Mg}^{+}$velocity distribution at several radial locations. Figure 2 depicts data and fits to typical $\mathrm{Mg}^{+}$distributions at three radial locations in the plasma. At larger radii, the velocity distributions are positively and negatively detuned from the $\mathrm{v}_{\theta}=0$ resonance because of a Doppler shift from the plasma rotation, either towards or away from the probe laser beam. This detuning is a direct measurement of the $E \times B$ rotation profile $\mathrm{v}_{\theta}(r)$. Radial profiles of the plasma temperature $T(r)$ and density of each $\mathrm{Mg}^{+}$isotope can also be obtained by fitting the velocity distribution at each radial location with Maxwellian distributions for each $\mathrm{Mg}^{+}$isotope. The relative density of the $\mathrm{Mg}^{+}$isotopes is determined from the relative area under each resulting Maxwellian fit.

Figure 3 shows the resulting measured density and rotation profiles (symbols) at three different rotation rates. These
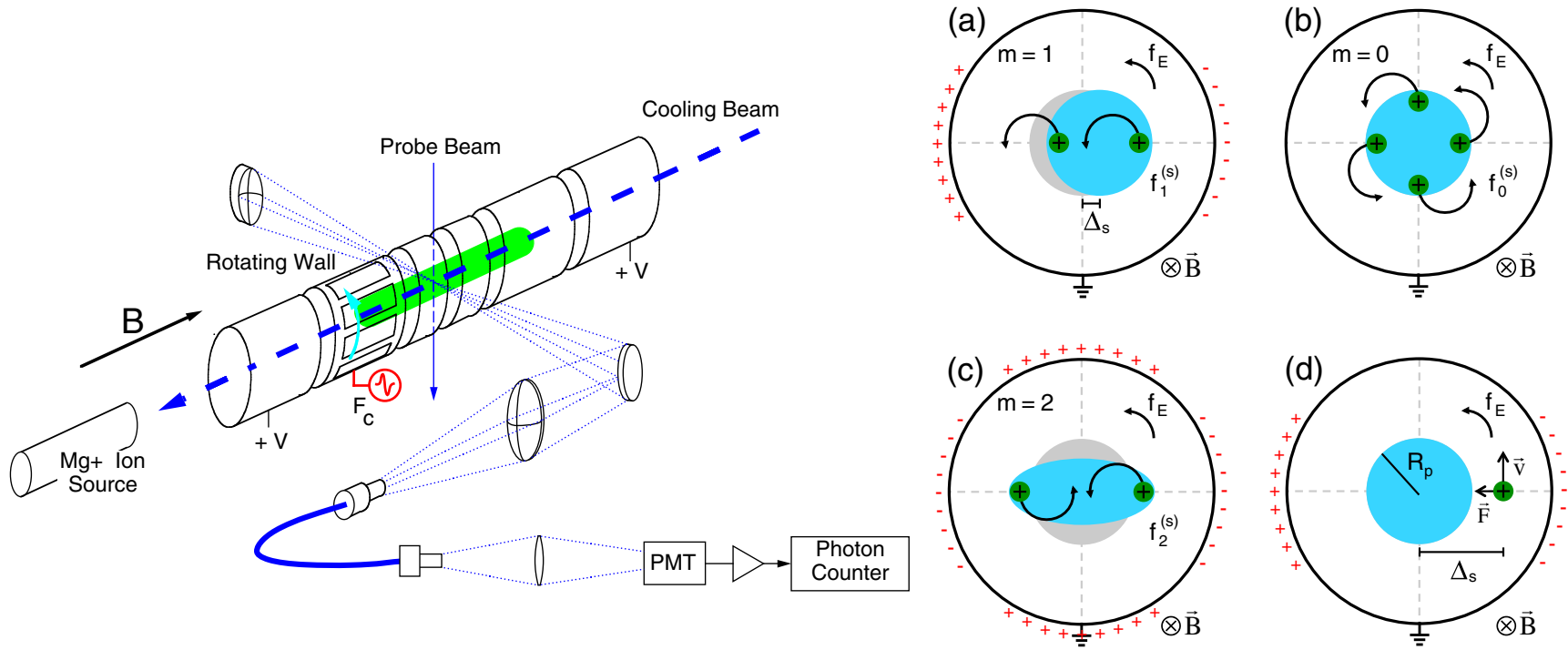

Figure 1. (Left) Schematic of a cylindrical Penning-Malmberg trap with "rotating wall" and laser diagnostics. (Right) Sketches of the $m=1, m=0$, and $m=2$ surface waves (a)-(c); and the large amplitude clump model (d) from an end view of the trap. The cyclotron mode frequencies are shifted proportional to the $E \times B$ drift-rotation frequency $f_{E}$, arising from trap potentials, image charges on the trap wall, and non-resonant species 


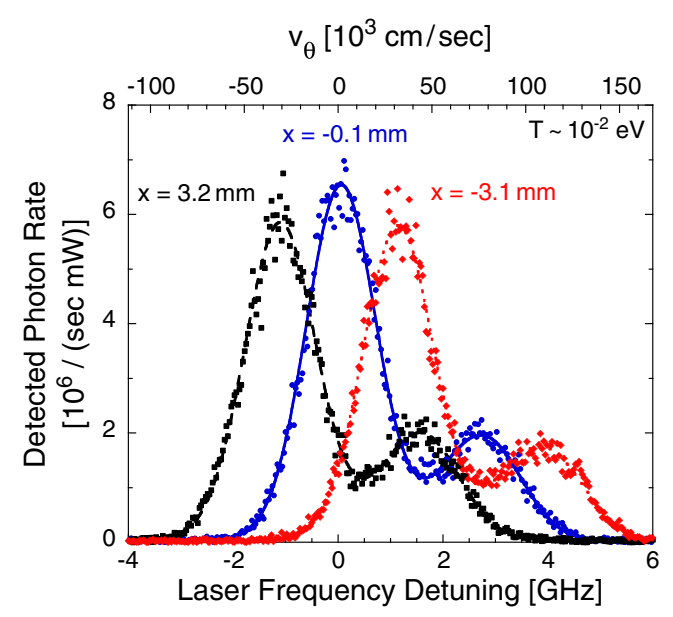

Figure 2. Data and fits of the LIF measured $\mathrm{Mg}^{+}$velocity distribution at three radial locations $r \equiv|x|$ in the plasma. At each location, the larger peak is the distribution of ${ }^{24} \mathrm{Mg}^{+}$, and the smaller hump is the combined distributions of ${ }^{25} \mathrm{Mg}^{+}$and ${ }^{26} \mathrm{Mg}^{+}$

profiles are a convolution of the true "top-hat" plasma profile with the finite size (half-width $0.39 \mathrm{~mm}$ ) probe laser beam. Obtaining the true plasma radius $R_{p}$ and rotation frequency $f_{E}$ is done by fitting to a convolved top-hat model with $n(r)=n_{0}$ and $\mathrm{v}_{\theta}(r)=2 \pi r f_{E}$ for $r \leq R_{p}$, giving dashed curves in Figure 3 profiles. The solid lines in Figure 3 are the resulting true "top-hat" $\mathrm{Mg}^{+}$density and rigid-rotor rotation profiles of the plasma. This rigid-rotor rotation frequency $f_{E}$ is a direct measurement of the total electric field strength from ion space charge and trap potentials.

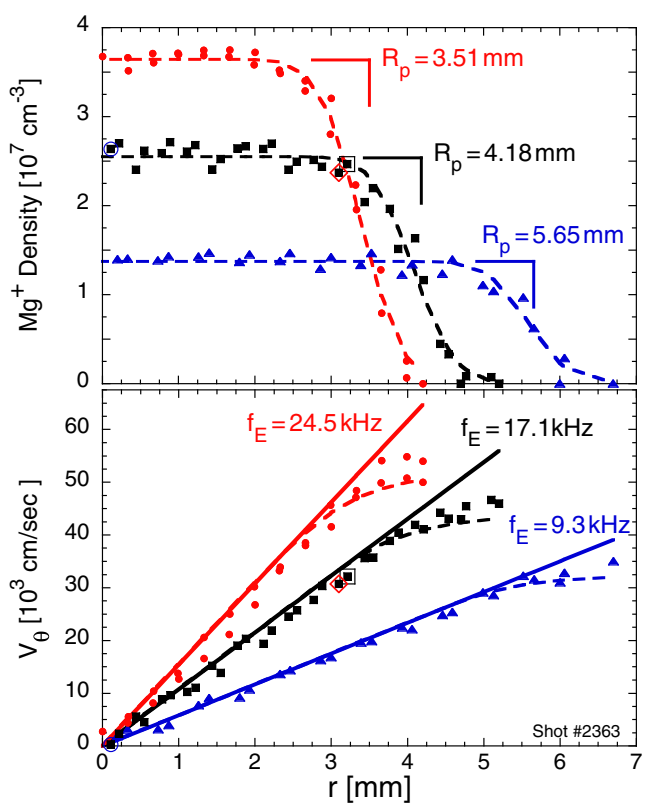

Figure 3. Radial profiles of $\mathrm{Mg}^{+}$density (top) and rotation velocities (bottom) at three different rotation rates. Symbols are laser-width-averaged data, and dashed curves are fits to the "top-hat" rigid-rotor model (solid lines). The three circled/ boxed data points correspond to the LIF signals of Figure 2
Laser cooling of the ${ }^{24} \mathrm{Mg}^{+}$ions enables temperature control; here, we maintain $T \sim 10^{-2} \mathrm{eV}=120 \mathrm{~K}$. In this regime, the ion clouds are radially uniform in density, with an abrupt edge, and the ion-ion collisionality $v_{i i} \sim 10^{3} / \mathrm{s}$ is moderate. At lower temperatures, the collisionality increases, and centrifugal separation of species occurs [15-17].

The cyclotron resonance frequencies are obtained by thermal cyclotron spectroscopy (TCS). A series of rf bursts, scanned over frequency, are applied to azimuthally sectored portions of the cylindrical wall, connected to create $\cos (m \theta)$ fields, with $m=1$ or 2 . For $m=0$, excitation is from the $\mathrm{z}$-end. The plasma is heated slightly when the rf burst is resonant with a particular mode at $f_{m}{ }^{(s)}$, and this is observed as a change in the laser cooling fluorescence. A cartoon of the TCS process is shown in the inset of Figure 4. Each species is excited individually with a burst of 10,000 cycles at wall amplitude $(0.1-3.0)$ $\mathrm{V}$, depending on the species and mode of interest. These excitations typically heat the plasma by approximately $10^{-2}$ $\mathrm{eV}$, corresponding to an amplitude of excitation $\Delta_{S} \sim 40 \mu \mathrm{m}$. The cyclotron excitation heats the perpendicular degree of freedom of species $s$, and this heat is transferred to other species and to the parallel degree of freedom through collisions $v_{\perp \|} \sim$ $700 / \mathrm{s}$, where it is removed/detected from ${ }^{24} \mathrm{Mg}^{+}$by the parallel cooling beam. TCS enables mode detection at small excitation amplitudes, $\Delta_{S}<<R_{w}$, which are below the noise floor for detection using induced charge on the conducting wall.

A typical broad $m=1$ TCS scan is shown in Figure 4. This TCS scan enables identification of the ion species, and the height of the peaks in Figure 4 (change in the cooling fluorescence $F$ ) depends on the relative species concentrations and on $\partial F / \partial T$. For short bursts, typically less than 200 cycles, we find

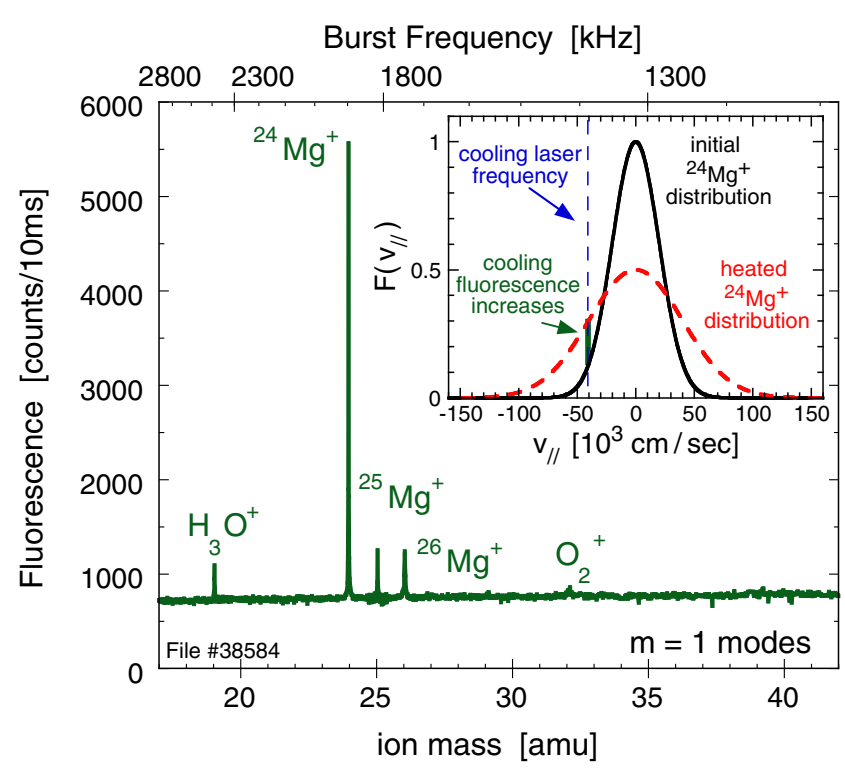

Figure 4. Mass spectra of a typical plasma containing ${ }^{24} \mathrm{Mg}^{+}$, ${ }^{25} \mathrm{Mg}^{+}$, and ${ }^{26} \mathrm{Mg}^{+}$, with $\mathrm{H}_{3} \mathrm{O}^{+}$and $\mathrm{O}_{2}{ }^{+}$impurity ions. The inset is a cartoon depicting the TCS process in which a cold $\mathrm{Mg}^{+}$ distribution is heated by the cyclotron motion, and this heat is detected as a change in the cooling laser fluorescence 
that the resultant plasma heating is proportional to the species concentrations $\delta_{s}$ as $\Delta T \propto \delta_{s} A_{B}{ }^{2} \tau_{B}{ }^{2} / M_{s}$, where $A_{B}$ is the burst amplitude and $\tau_{B}$ is the burst period. The relative $\mathrm{Mg}^{+}$ratio $\delta_{26} /$ $\delta_{24}$ has been measured using this short burst heating technique and is found to be within $10 \%$ of that obtained from LIF diagnostics. However, throughout this work, we have used bursts of 10,000 cycles for better frequency resolution. In this regime, the plasma heating "saturates," most likely because of ion-ion collisions that dephase the cyclotron mode from the drive.

\section{Results}

Frequency shifts are measured for the $\mathrm{m}=0,1$, and 2 cyclotron modes, having density perturbations varying as $\delta n \propto \cos (m \theta-$ $\left.2 \pi f_{m}{ }^{(s)} t\right)$. The $m=1$ mode can be thought of as a surface wave, or as the small amplitude equivalent of the center-of-mass mode used in FTICR-MS experiments. This mode, driven using a dipole burst, corresponds to a displacement of the excited species off axis, with the center-of-mass of that single species orbiting the center of the trap at a frequency near $f_{1}^{(s)}$, as depicted in Figure 1a. In contrast, the $m=0$ mode is a radial "breathing" of the plasma cross-section as shown in Figure $1 \mathrm{~b}$. It generates no external electric field except at the plasma ends. To excite this mode, the end of the plasma is wiggled at $f_{0}^{(s)}$. The $m=2$ mode is an elliptical deformation of the cross section of that species, giving a wall signal at $f_{2}{ }^{(s)}$, as in Figure 1c. This mode is excited using a quadrupole burst, i.e., $90^{\circ}$ wall sectors phased as $+/-1+/-$.

Figure 5 (left) shows the $\mathrm{m}=0,1$, and 2 cyclotron mode frequencies for ${ }^{24} \mathrm{Mg}^{+}$. It can be seen that the $m=0$ and $m=1$ modes are downshifted from the "bare" cyclotron frequency $F_{c}^{(24)}=1899.46 \mathrm{kHz}$, whereas the $m=2$ mode is upshifted. The difference in frequency between these modes closely equals the measured $E \times B$ rotation frequency $f_{E}=9.3 \mathrm{kHz}$.

The rotating wall allows us to compress the plasma, obtaining cyclotron mode frequencies at different $f_{E}$ for the same trapped ions. In Figure 5 (right) the resonant frequency of the $m=1$ cyclotron mode is shown for three values of $f_{E}$. The downshift of the $m=1$ cyclotron frequency increases as the ion density is increased, because of the increased $f_{E}$.

Plotted in Figure 6 are $\mathrm{m}=0,1$, and 2 cyclotron frequencies versus $f_{E}$ for ${ }^{24} \mathrm{Mg}^{+},{ }^{25} \mathrm{Mg}^{+}$, and ${ }^{26} \mathrm{Mg}^{+}$. The shift of the cyclotron frequency increases linearly as space charge is increased, as seen in previous experiments[18-21]. However, this increase with $f_{E}$ is also proportional to the relative charge concentration $\delta_{s}$ of the species. The proportionality constants are different for the majority species ${ }^{24} \mathrm{Mg}^{+}$than for the minority species ${ }^{25} \mathrm{Mg}^{+}$and ${ }^{26} \mathrm{Mg}^{+}$, because of plasma collective effects. These experimental results clearly show that the frequency shifts are dependent on the total charge density, giving rise to $f_{E}$, and on the relative charge concentration of the species $\delta_{s}$, but further specificity requires theory.

\section{Theory}

\section{Surface Wave}

In the limit of small excitation amplitude $\Delta_{s}<R_{p}$, these cyclotron modes are best treated as plasma surface waves. The "guiding center" of each ion $E \times B$ drifts around the trap center at constant radius $r<R_{p}$, with cyclotron excitation causing superimposed cyclotron orbits of radius $r_{c} \sim \Delta_{s}<R_{p}$. For the $m=1$ mode, the cyclotron orbits are all in phase, causing the species center-of-mass to orbit the trap center at $r \sim \Delta_{s}$ with frequency $f_{1}^{(s)}$ (Figure 1a). In contrast, the ion orbits for the $m=$ 0 and $m=2$ modes are phased such that radially "breathing" and elliptical density perturbations are formed (Figure $1 \mathrm{~b}$ and c).

The cyclotron mode frequency is obtained by solving the Vlasov-Poisson equation for an $m$ perturbation in a frame rotating with the plasma at $f_{E}^{S C}[6-8]$. The resulting lab-frame frequency is given by Equation 1, if trap electric fields are negligible. The $\delta_{s}\left(1-\mathscr{R}_{m}\right) f_{E}^{S C}$ term represents collective interactions in the plasma frame rotating at $f_{E}{ }^{S C}$; the $-2 f_{E}^{S C}$ term is the Coriolis force in the plasma frame; and the $m f_{E}{ }^{S C}$ term is the Doppler shift back to the lab frame.

We note that the radial electric field of the trap potentials will result in an increased $E \times B$ rotation frequency $f_{E}$ causing additional frequency shifts as

$f_{m}^{(s)}-F_{c}^{(s)}=\left[(m-2)+\delta_{s}\left(1-\mathscr{R}_{m}\right)\right] f_{E}^{S C}-(m-2) f_{E}^{T}$,

although this result has not been rigorously derived. Here, $f_{E}^{T}$ is the $E \times B$ rotation frequency resulting from the trap potentials. In these experiments, image charge and trap potentials cause frequency shifts of about 100 and $50 \mathrm{~Hz}$, respectively. The surface wave theory includes image charge corrections through $\mathscr{R}_{m}$, but does not include the finite length effects[22] resulting from the trap potentials. Ignoring this shift, we find that this surface wave theory predicts that the cyclotron modes depend on $f_{E}$, and on the relative fraction of the species $\delta_{s}$, as seen in the experiments. It also predicts the observed $f_{E}$ spacing between the cyclotron modes when $\mathscr{R}_{m}<<1$.

Fitting Equation 1 to the measured frequency shifts in Figure 6, we find that the observed mode frequency spacing is consistent to within the $2 \%$ accuracy of the LIF measurement of $f_{E}$, and that these cyclotron modes converge to the "bare" cyclotron frequency $F_{c}^{(s)}$ in the limit $f_{E} \rightarrow 0$. Also, the slope of the frequency shifts in Figure 6 provide a measurement of the charge fraction $\delta_{s}$ for each species. These agree to within $20 \%$ with the species density ratios $\delta_{25} / \delta_{24}$ and $\delta_{26} / \delta_{24}$ obtained directly from LIF measurements, which is less well calibrated in absolute densities. The corresponding mass ratios from $F_{c}^{(s)}$ are accurate to within $200 \mathrm{ppm}$.

Absent laser diagnostics, four frequencies from two m-theta modes in two plasma states could be used to determine the plasma characteristics $f_{E}$ and $\delta_{s}$, and thereby determine $F_{c}{ }^{(s)}$. In Figure 6, the measured cyclotron frequency differences of the two circled (vertical) data pairs give $f_{E}=(9.33,16.97) \mathrm{kHz}$ versus the measured $(9.29,17.13) \mathrm{kHz}$; Equation 1 then gives 

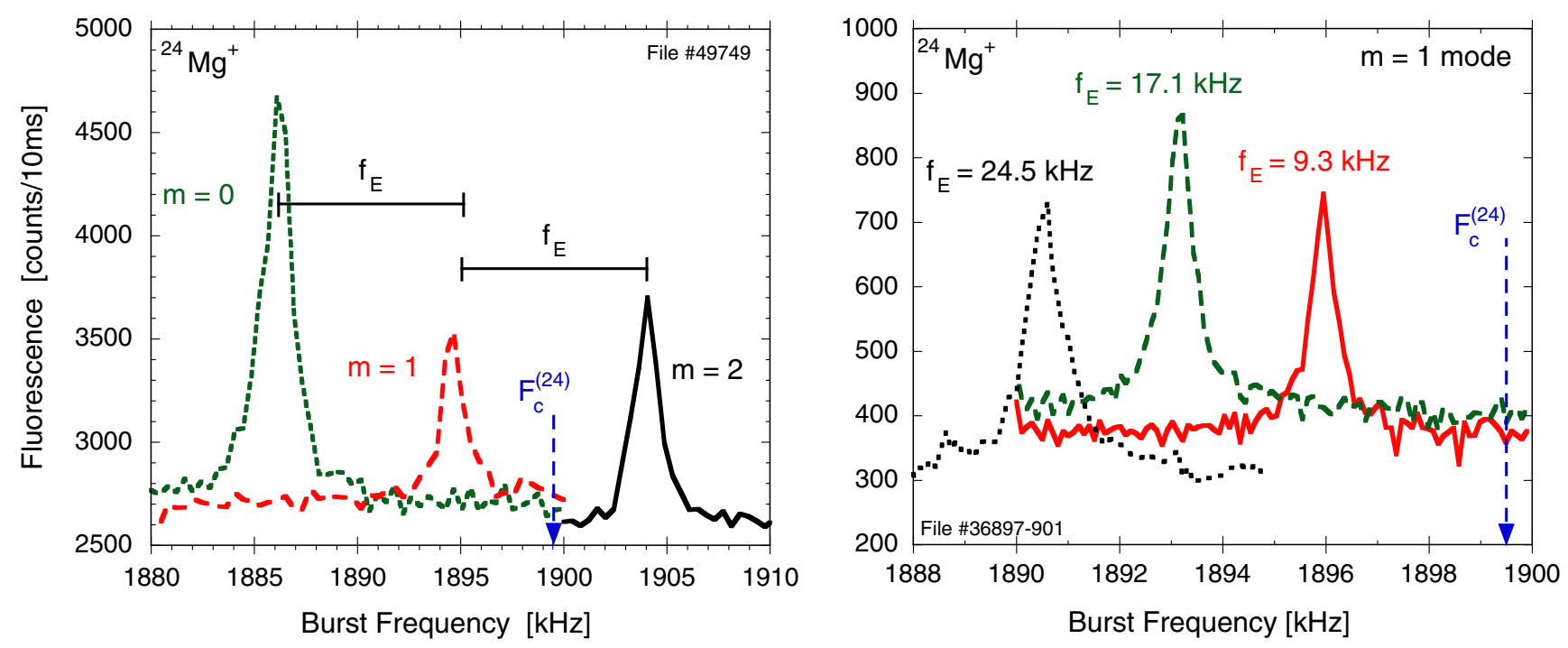

Figure 5. (Left) Observed ${ }^{24} \mathrm{Mg}^{+}$cyclotron resonances for $\mathrm{m}=0,1$, and 2 modes. Modes are shifted away from the "bare" cyclotron frequency $F_{c}{ }^{(24)}=1899.22 \mathrm{kHz}$, and have a frequency spacing of the $E \times B$ rotation frequency $f_{E}=9.3 \mathrm{kHz}$. (Right) Measured $m=1$ cyclotron resonance for ${ }^{24} \mathrm{Mg}^{+}$at three different rotation rates. As $f_{E}$ is increased, through an increase in the plasma density, the $m=1$ cyclotron mode frequency shift increases

$\delta_{26}=9.06 \%$ and $F_{c}{ }^{(s)}=1753.82 \mathrm{kHz}$, in close agreement with the full data results in Figure 6. Of course, similar information from multiple species would improve this plasma characterization.

\section{Clump Model}

In FTICR-MS, the $m=1$ cyclotron mode is generally modeled as a clump of charge orbiting around the trap center at radius $\Delta_{s}$, as shown in Figure 1d. This center-of-mass motion is equivalent to the $m=1$ surface wave in the limit $\Delta_{s}<R_{p}$. This clump model will be used in this section in order to better understand the physics of the cyclotron frequency shift, and to produce the calibration equation, Equation 2.

In this clump model, the equilibrium ion cloud is treated as a long centered cylinder with radius $R_{p}$, consisting of multiple species each with uniform density $n_{s}$, and a total density $n_{0}=\sum n_{s}$. The center-of-mass of one species is then displaced uniformly by $\Delta_{s}$, and it orbits the trap center with velocity $\mathrm{v}_{s}$, giving mode frequency $f_{1}^{(s)}=\mathrm{v}_{s} / 2 \pi \Delta_{s}$. The radial forces acting on the center-of-mass of the displaced clump are the centrifugal, $\mathrm{v}_{s} \times B$, and electrostatic forces, summing to zero as

$\frac{M_{s} \mathrm{v}_{s}^{2}}{\Delta_{s}}-q_{s} \mathrm{v}_{s} B_{z}+q_{s} \widetilde{E}_{r}=0$.

Here, $\tilde{E}_{r}$ represents the electric field of the non-resonant species, trap potentials, and image charge of the resonant species, rather than the total electric field $E_{r}$ measured through $f_{E}$. Equation 4 reduces to $f_{1}^{(s)}=F_{c}^{(s)}$ when there is no electrostatic force $\tilde{E}_{r}=0$. When a radial electric field is present, the cyclotron mode frequency is shifted away from the "bare" cyclotron frequency, and Equation 4 reduces to the $m=1$ result of Equation 3 for small $\tilde{E}_{r}$.
The electric field arising directly from species $s$ charge does not affect the center-of-mass motion of species $s$. In a "point clump" model this is obvious; for a finite-size clump the relevant spatial integrals give the same result. However, the wall image charge for species $s$ does affect the orbital dynamics of species $s$ clump. Even with only one species, the wall image charge affects orbital dynamics, commonly exemplified in the low-frequency $E \times B$ drifting "diocotron" mode[5].

Solving for $(M / q)_{s}$ in Equation 4 produces a calibration equation,

$\left(\frac{M}{q}\right)_{s}=\frac{B_{\mathrm{z}}}{2 \pi f_{1}^{(s)}}-\frac{1}{\left(2 \pi f_{1}^{(s)}\right)^{2}} \frac{\widetilde{E}_{r}}{\Delta_{s}}$.

The accuracy of Equation 5 depends on correctly modeling $\widetilde{E}_{r} / \Delta_{S}$ at the radial position $\Delta_{S}$ of the clump. Note that $E_{r} / r$ represents the $E \times B$ drift rotation frequency, as $f_{E}(r)=\left(E_{r} / r\right) /$ $2 \pi B$. In our experiments $f_{E}(r)$ is uniform with $\mathrm{r}$.

When the equilibrium charge densities are uniform and the excitation amplitude is small (i.e., $\Delta_{s}<R_{p}$ ), the electric field ratio $\tilde{E}_{r} / \Delta_{s}$ is basically independent of radius. Then, the "calibration" of Equation 5 is independent of radius. The ion cloud is also assumed to be long compared with its radius $L_{p}>>R_{p}$, so that the ion cloud and image charge electric fields can be approximated as those of an infinite cylinder. The partial electric field $\tilde{E}_{r}$ produced from the non-excited ion cloud species, from image charges of the excited species, and from trap potentials is then

$\frac{\widetilde{E}_{r}}{\Delta_{s}}=\left(1-\delta_{s}\right) \frac{e n_{0}}{2 \epsilon_{0}}+\delta_{s} \frac{e n_{0}}{2 \epsilon_{0}} \mathscr{R}_{1}+2 V_{T} G_{T}$ 


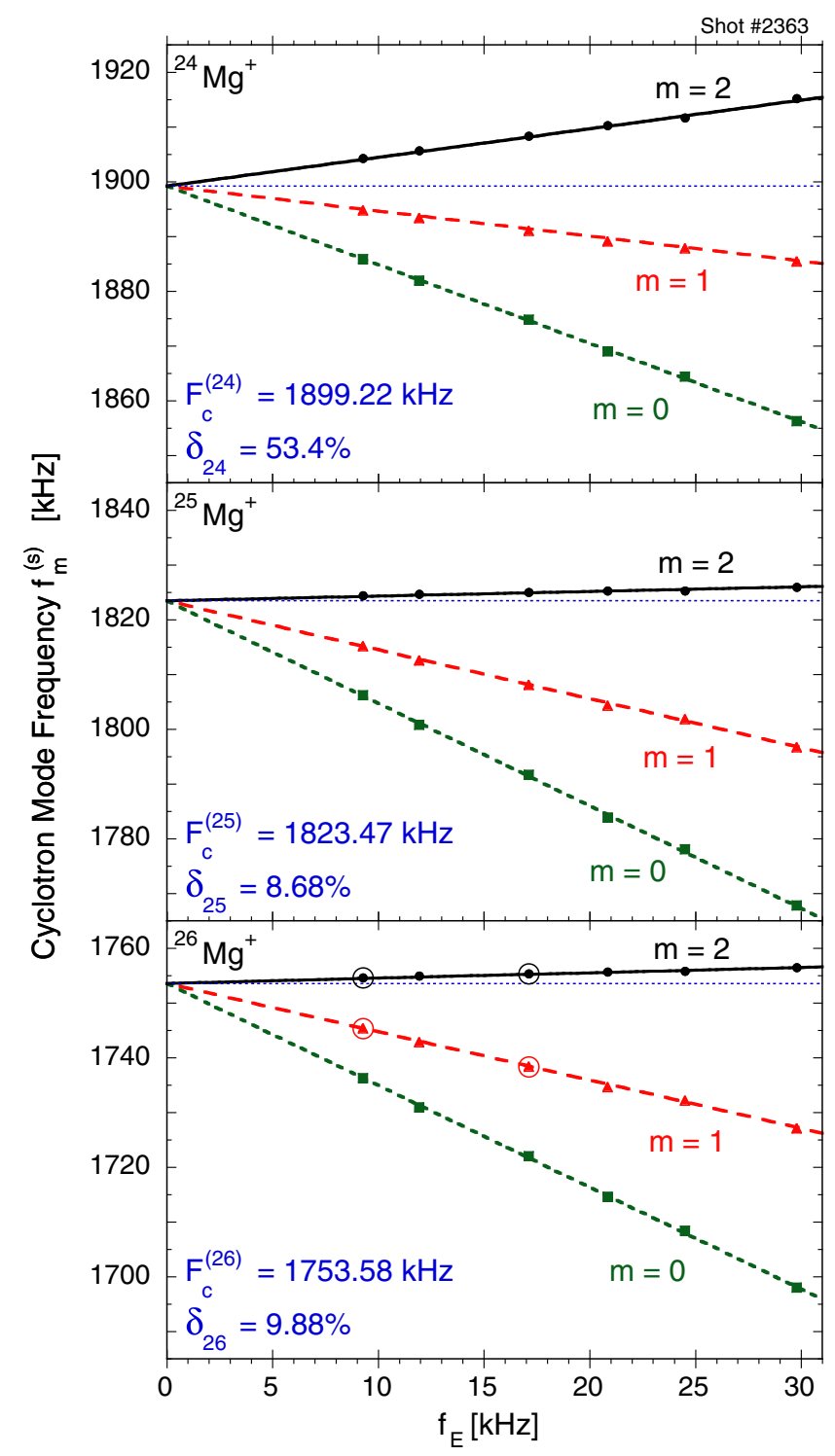

Figure 6. Cyclotron mode frequencies versus $f_{E}$ for ${ }^{24} \mathrm{Mg}^{+}$, ${ }^{25} \mathrm{Mg}^{+}$, and ${ }^{26} \mathrm{Mg}^{+}$. Symbols are experimental data and curves are fits to Equation 1, which determine $F_{c}{ }^{(s)}$ (dotted) and $\delta_{s}$ for each species

where $e n_{0}=Q_{0} / \pi R_{p}^{2} L_{p}$ is the total charge density, and $G_{T}$ is a geometrical factor for the trap shape[23].

Here, we see that the dependence on $\delta_{s}$ comes from the reduction of the effective electric field, since the excited species cannot apply a force on itself; and from the image charge in the confining wall, which does apply a force on the charge clump. The image charges of the non-excited species are $\theta$-symmetric, and the image charges of the other excited species are assumed to be non-resonant and, therefore, time-average to zero. By taking $\delta_{s} \rightarrow 0$, (i.e., treating a single particle excitation and ignoring image charge) this $\tilde{E}_{r}$ electric field reduces to that of Jeffries et al.[23].
From this $\tilde{E}_{r} / \Delta_{s}$ a calibration equation can be obtained,

$$
\begin{aligned}
\left(\frac{M}{q}\right)_{s}= & \overbrace{B_{z}}^{A} \frac{1}{2 \pi} \frac{1}{f_{1}^{(s)}} \\
& +[\overbrace{-\frac{1}{(2 \pi)^{2}}\left(\frac{e n_{0}}{2 \varepsilon_{0}}+2 V_{T} G_{T}\right)}^{B}+\overbrace{\frac{e n_{0}}{2 \varepsilon_{0}}\left\{\frac{1-\mathscr{R}_{1}}{(2 \pi)^{2}}\right\}}^{C} \delta_{s}] \frac{1}{\left(f_{1}^{(s)}\right)^{2}} .
\end{aligned}
$$

This calibration equation depends on the relative charge density $\delta_{s}$ of species $s$. If the excitation amplitudes $\Delta_{s}$ are the same for each species, the received wall signal $I_{s}$ is a measure of $\delta_{s}$, and Equation 7 results in the "intensity-dependent" calibration equation of references $[10,11]$. In general, $I_{S}$ is only an approximate measure of $\delta_{s}$, since it is also proportional to the excitation amplitude, as $I_{s} \propto \delta_{s} \Delta_{s}$. The parameters $A$ and $B$ are identical to those of the simple calibration equation[24]. They calibrate single particle effects such as the magnetic field strength and frequency shifts from the entire ion cloud and trap potentials. Parameter $C$ corrects for overestimating the frequency shift from the ion cloud when self-forces between the excited species and itself were included, and also includes the effects of image charge.

\section{Conclusion}

The simplest calibration equation, Equation 2 with $C=0$, overestimates the downshift in the cyclotron frequency by assuming that the excited species can exert a force on itself. With proper forces, the cyclotron frequency shifts of a multi-species ion cloud are dependent on the relative concentrations of the species. This has been demonstrated experimentally here by showing that the shift of the $m=1$ cyclotron frequency is larger for the minority species, and, theoretically, by presenting a surface wave model that is in quantitative agreement with our experiments. These surface wave results have been interpreted through a clump model, which correctly predicts the "intensity-dependent" calibration equation that has been shown to improve mass accuracies in FTICR-MS experiments.

Cyclotron modes with different $\cos (m \theta)$ dependence have also been presented. The frequency spacing between these cyclotron modes with different $m$ can provide a measurement of the electric field strength. By exciting these modes in FTICR-MS experiments, limits can be placed on the size of the cyclotron frequency shifts. The effects of space charge can also be reduced by varying the electric field strength and extrapolating the $\mathrm{m}=0$, 1 , and 2 mode frequencies to the "bare" cyclotron frequency.

\section{Acknowledgments}

The authors acknowledge support for this work by National Science Foundation grant PHY-1414570, and Department of Energy grants DE-SC0002451 and DE-SC0008693. 


\section{References}

1. Zhang, L.-K., Rempel, D., Pramanik, B.N., Gross, M.L.: Accurate mass measurements by Fourier transform mass spectrometry. Mass Spectrom. Rev. 24(2), 286-309 (2005)

2. Barlow, S., Luine, J., Dunn, G.: Measurement of ion/molecule reactions between 10 and 20 k. Int. J. Mass Spectrom. Ion Process 74(1), 97-128 (1986)

3. Rempel, D., Ledford Jr., E., Huang, S., Gross, M.: Parametric mode operation of a hyperbolic penning trap for Fourier transform mass spectrometry. Anal. Chem. 59(20), 2527-2532 (1987)

4. Peurrung, A., Kouzes, R., Barlow, S.: The non-neutral plasma: an introduction to physics with relevance to cyclotron resonance mass spectrometry. Int. J. Mass Spectrom. Ion Process 157, 39-83 (1996)

5. Gould, R.W., LaPointe, M.A.: Cyclotron resonance in a pure electron plasma column. Phys. Rev. Lett. 67, 3685-3688 (1991)

6. Sarid, E., Anderegg, F., Driscoll, C.F.: Cyclotron resonance phenomena in a non-neutral multispecies ion plasma. Phys. Plasmas 2(8), 2895-2907 (1995)

7. Gould, R.W.: Theory of cyclotron resonance in a cylindrical non-neutral plasma. Phys. Plasmas 2(5), 1404-1411 (1995)

8. Dubin, D.H.: Cyclotron waves in a non-neutral plasma column. Phys. Plasmas 20, 042120 (2013)

9. Affolter, M., Anderegg, F., Dubin, D., Driscoll, C.: Cyclotron mode frequency shifts in multi-species ion plasmas. Phys. Lett. A 378(3233), 24062409 (2014)

10. Burton, R., Matuszak, K., Watson, C., Eyler, J.: Exact mass measurements using a 7 tesla Fourier transform ion cyclotron resonance mass spectrometer in a good laboratory practices-regulated environment. J. Am. Soc. Mass Spectrom. 10(12), 1291-1297 (1999)

11. Masselon, C., Tolmachev, A., Anderson, G., Harkewicz, R., Smith, R.: Mass measurement errors caused by local frequency perturbations in FTICR mass spectrometry. J. Am. Soc. Mass Spectrom. 13(1), 99-106 (2002)

12. Savory, J., Kaiser, N., McKenna, A., Xian, F., Blakney, G., Rodgers, R., Hendrickson, A., Marshall, C.: Parts-per-billion Fourier transform ion cyclotron resonance mass measurement accuracy with a walking calibration equation. Anal. Chem. 83(5), 1732-1736 (2011)

13. Anderegg, F., Hollmann, E., Driscoll, C.: Rotating field confinement of pure electron plasmas using Trivelpiece-Gould modes. Phys. Rev. Lett. 81(22), 4875-4878 (1998)
14. Anderegg, F., Huang, X.-P., Sarid, E., Driscoll, C.F.: A new pure ion plasma device with laser induced fluorescence diagnostic. Rev. Sci. Instrum. 68(6), 2367-2377 (1997)

15. O'Neil, T.: Centrifugal separation of a multispecies pure ion plasma. Phys. Fluids 24, 1447-1451 (1981)

16. Larson, D., Bergquist, J., Bollinger, J., Itano, W.M., Wineland, D.: Sympathetic cooling of trapped ions: a laser-cooled two-species nonneutral ion plasma. Phys. Rev. Lett. 57(1), 70-73 (1986)

17. Andresen, G. B., Ashkezari, M. D., Baquero-Ruiz, M., Bertsche, W., Bowe, P.D., Butler, E., Cesar, C.L., Chapman, S., Charlton, M., Deller, A.Eriksson, S., Fajans, J., Friesen, T., Fujiwara, M. C., Gill, D. R., Gutierrez, A., Hangst, J. S., Hardy, W. N., Hayden, M. E., Humphries, A. J.,Hydomako, R., Jonsell, S., Madsen, N., Menary, S., Nolan, P., Olin, A., Povilus, A., Pusa, P., Robicheaux, F.,Sarid, E., Silveira, D. M., So, C., Storey, J. W., Thompson, R. I., van der Werf, D. P., Wurtele, J. S., Yamazaki,Y.: Centrifugal separation and equilibration dynamics in an electron-antiproton plasma. Phys. Rev. Lett. 106(14), 145001 (2011)

18. Easterling, M., Mize, T., Amster, I.: Routine part-per-million mass accuracy for high-mass ions: space-charge effects in MALDI FT-ICR. Anal. Chem. 71(3), 624-632 (1999)

19. Van Dyck Jr., R., Moore, F., Farnham, D., Schwinberg, P.: Number dependency in the compensated penning trap. Phys. Rev. A 40(11), 6308-6313 (1989)

20. Taylor, P., Amster, I.: Space charge effects on mass accuracy for multiply charged ions in ESI-FTICR. Int. J. Mass Spectrom. 222(1), 351-361 (2003)

21. Wong, R., Amster, I.: Experimental evidence for space-charge effects between ions of the same mass-to-charge in Fourier-transform ion cyclotron resonance mass spectrometry. Int. J. Mass Spectrom. 265(2), 99-105 (2007)

22. Fine, K.S., Driscoll, C.F.: The finite length diocotron mode. Phys. Plasmas 5(3), 601-607 (1998)

23. Jeffries, J., Barlow, S., Dunn, G.: Theory of space-charge shift of ion cyclotron resonance frequencies. Int. J. Mass Spectrom. Ion Process 54(1), 169-187 (1983)

24. Ledford, E.B., Rempel, D.L., Gross, M.L.: Space charge effects in Fourier transform mass spectrometry II. Mass calibration. Anal. Chem. 56(14), 2744-2748 (1984) 